\title{
Um Algoritmo Eficiente para o Problema do Posicionamento Natural de Antenas *
}

\author{
Bruno E. Crepaldi ${ }^{1}$, Cid C. de Souza ${ }^{1}$, Pedro J. de Rezende ${ }^{1}$ \\ ${ }^{1}$ Instituto de Computação - Universidade Estadual de Campinas (UNICAMP) \\ brunoecrepaldi@gmail.com, rezende@ic.unicamp.br, cideic.unicamp.br
}

\begin{abstract}
Considered a variation of the art gallery problem, the wireless localization problem deals with the placement of the smallest number of broadcasting antennas required to determine if a person is inside or outside the gallery. Each antenna propagates a unique key within a certain antenna-specific angle of broadcast, and the set of keys received at any given point must be sufficient to determine whether that point belongs or not to the polygon representing the gallery. To ascertain this localization property, a Boolean formula must be produced along with the placement of the antennas. In this thesis, we present an exact algorithm based on integer linear programming and geometrical properties for solving the natural wireless localization problem, which is known to be $\mathbb{N P}$-hard. The efficiency of the algorithm is certified by experimental results comprising the solutions of 720 instances, including polygon with holes with up to 1000 vertices, in less than six minutes on a standard desktop computer.
\end{abstract}

Resumo. Considerado uma variação do problema da galeria de arte, o problema do posicionamento de antenas trata da localização do menor número de antenas requerido para determinar se uma pessoa está dentro ou fora da galeria. Cada antena propaga uma chave única dentro de um ângulo específico de transmissão, e o conjunto de chaves recebidas em um dado ponto deve ser suficiente para decidir se ele pertence ou não ao polígono que representa a galeria. Para verificar esta propriedade de localização, uma fórmula Booleana deve ser produzida junto com o posicionamento das antenas. O problema do posicionamento natural de antenas é $\mathbb{N P}$-difícil. Nesta dissertação, apresentamos um algoritmo exato baseado em programação linear inteira e propriedades geométricas para resolvê-lo. A eficiência do algoritmo é certificada por resultados experimentais que compreendem as soluções ótimas de 720 instâncias, incluindo polígono com buracos de até 1000 vértices, calculadas em menos de seis minutos em um computador desktop padrão.

\section{Introdução}

Problemas de Galeria de Arte [O'Rourke 1987, Urrutia 2000, Couto et al. 2011, Borrmann et al. 2013] compõem um tema de pesquisa de longa data em Geometria Computacional. Variantes do problema surgiram após a introdução de um novo conceito

\footnotetext{
${ }^{*}$ Esta pesquisa teve apoio de CNPQ (Proc. \#311140/2014-9, \#477692/2012-5 e \#302804/2010-2), FAPESP (Proc. \#2007/52015-0 e \#2012/17608-9) e FAEPEX/UNICAMP.
} 

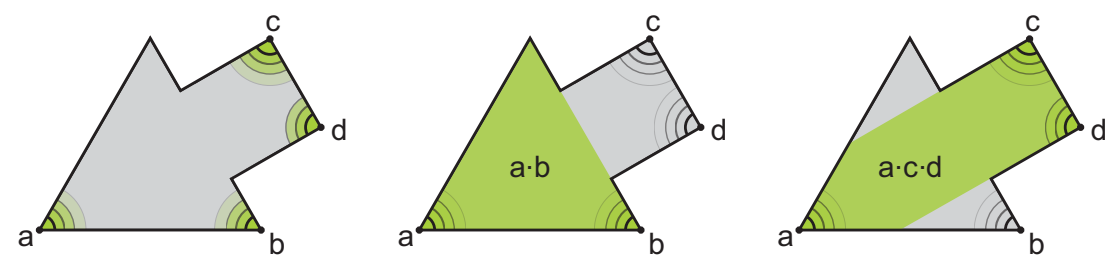

Figura 1. Exemplo com antenas em $a, b, c$ e $d$ e fórmula Booleana a.b+a.c.d.

de visibilidade, em que os guardas são capazes de ver através das paredes da galeria [Eppstein et al. 2007]. A motivação para esta modificação origina-se a partir de aplicações para redes sem-fio, onde os sinais das antenas não são bloqueados pelas paredes das construções. É nesse contexto que surge o Problema do Posicionamento de Antenas (WLP, Wireless Localization Problem).

Para ilustrar o WLP, considere o seguinte exemplo, que captura a essência do problema [Christ and Hoffmann 2009]. O proprietário de um cybercafé gostaria de fornecer internet sem-fio aos seus clientes, mas quer evitar que aqueles que estão fora da loja possam acessar sua infra-estrutura de rede. Para conseguir isso, antenas podem ser instaladas, cada uma das quais transmitindo uma chave única (secreta) num ângulo arbitrário fixo. $\mathrm{O}$ objetivo é instalar essas antenas e ajustar seus ângulos de transmissão de tal forma que os clientes dentro da área do cybercafé possam ser distinguidos daqueles fora da loja simplesmente pedindo para nomearem quais as chaves que são recebidas no local onde se encontram. Para verificar sua localização, um cliente faz uso de uma fórmula Booleana cujas variáveis correspondem às chaves transmitidas, cada uma delas tendo, respectivamente, o valor verdadeiro ou falso dependendo se, no ponto onde se encontra, o cliente recebe ou não aquela chave. Uma vez que a instalação e manutenção das antenas têm um custo, um problema de otimização natural, é o de encontrar uma solução com o número mínimo de tais dispositivos. As semelhanças entre este problema e o da galeria de arte são evidentes. Assim, a região de interesse é uma loja (cybercafé) ao invés de uma galeria, e os guardas deste último são antenas no primeiro. Uma instância do problema pode ser vista na Figura 1. Neste caso, uma solução ótima seria instalar antenas nos vértices $a, b, c$ e $d$, com ângulo de transmissão idêntico ao ângulo interno do polígono nesses vértices, e usar a fórmula Booleana dada por $a \cdot b+a \cdot c \cdot d$.

Uma forma alternativa de visualizar o problema é observar que a área de transmissão de uma antena é um cone ilimitado no plano e que o polígono correspondente à área da loja pode ser definido por meio de uniões e intersecções desses cones. Com isto, a fórmula Booleana procurada pode ser vista como as uniões e intersecções necessárias, onde cada variável representa um cone. Uma aplicação direta do problema decorrente desta visão geométrica, é sua utilização em Geometria Sólida Construtiva [Dey 1991], uma técnica utilizada na área de modelagem de sólidos.

A pesquisa nesta dissertação foi focada na variante do WLP chamada de Problema do Posicionamento Natural de Antenas, onde as antenas estão limitadas a estarem nos vértices e arestas do polígono e com ângulo de transmissão idêntico ao ângulo interno naquele ponto. Apesar dos vários resultados teóricos publicados sobre o problema [Dobkin et al. 1993, Eppstein et al. 2007, Christ et al. 2008, Christ et al. 2009], 
anteriormente ao nosso trabalho, não havia na literatura propostas de algoritmos de qualquer natureza (exato ou heurístico) para resolvê-lo. Assim, como era de se esperar, tão pouco existiam instâncias de domínio público para serem testadas experimentalmente. A partir destas observações, o preenchimento destas duas lacunas foi fixado como sendo o objetivo do trabalho.

Além disso, por ser tratar de um problema na área de Geometria Computacional, onde estudos algorítmicos de problemas $\mathbb{N P}$-difíceis tendem a se iniciar diretamente com heurísticas e aproximações, optou-se por buscar um algoritmo exato para resolvê-lo baseado em programação linear inteira (PLI). A razão desta escolha foram os ótimos resultados obtidos para problemas geométricos de galeria de arte [Couto et al. 2008, Couto et al. 2011, Borrmann et al. 2013] com o emprego desta mesma metodologia.

Nesta dissertação, a partir de uma discretização para o problema do posicionamento natural de antenas, apresentamos um modelo PLI capaz de resolvê-lo. A partir do modelo inicial, conduzimos uma série de aperfeiçoamentos matemáticos e aplicações de propriedades geométricas para otimizá-lo. A cada mudança, efetuamos uma avaliação experimental do algoritmo, com o intuito não apenas de mostrar os resultados alcançados mas, também, de apontar as limitações práticas que nos levaram a sugerir as alterações propostas na sequência.

Como produto final desta pesquisa, foi obtido um algoritmo iterativo capaz de resolver instâncias não triviais do problema na otimalidade. Os resultados da dissertação deram origem a duas publicações internacionais, sendo uma em conferência [Crepaldi et al. 2013a] e outra em um periódico [Crepaldi et al. 2015]. Note-se que tanto a conferência quanto o periódico são bastante conceituados junto à comunidade da área de Geometria Computacional. Além disso, um benchmark contendo todas as instâncias testadas e seus resultados foram disponibilizados publicamente [Crepaldi et al. 2013b].

\section{Ideia Geral da Solução}

No WLP, uma antena pode ser vista como uma estação, posicionada numa dada localização, que transmite um sinal num ângulo e direção predefinidos. A região coberta por uma antena localizada no ponto $p$ é o cone com ápice em $p$ definido por dois raios emanando deste ponto. Estes dois raios definem o ângulo e a direção de transmissão. Assim, de uma maneira mais formal, a região de transmissão de uma antena é um cone ilimitado no plano definido pela intersecção ou união de no máximo dois meios-planos.

Podemos associar a uma antena $g$ uma variável Booleana que, para cada ponto $p$ no plano, tem um valor verdadeiro se e somente se $p$ está contido no cone de transmissão de $g$. Então, dado um polígono $P$ e um conjunto de antenas $G$, deseja-se saber se existe uma fórmula Booleana $B$, na forma normal disjuntiva, com estas variáveis, que é satisfeita exclusivamente pelos pontos de $P$. Caso afirmativo, diz-se que $G$ é capaz de descrever $P$.

No contexto do problema, é dado um polígono $P$ e um conjunto $G$ de potenciais antenas, cada uma delas caracterizada pela sua localização e por seu ângulo de transmissão, capazes de descrever $P$. Quando um custo unitário é atribuído a cada antena de $G$, o problema de otimização procura um subconjunto de $G$ que descreve $P$ e que tenha o menor custo.

Podemos obter uma subdivisão planar induzida pelo cone de transmissão de todas 


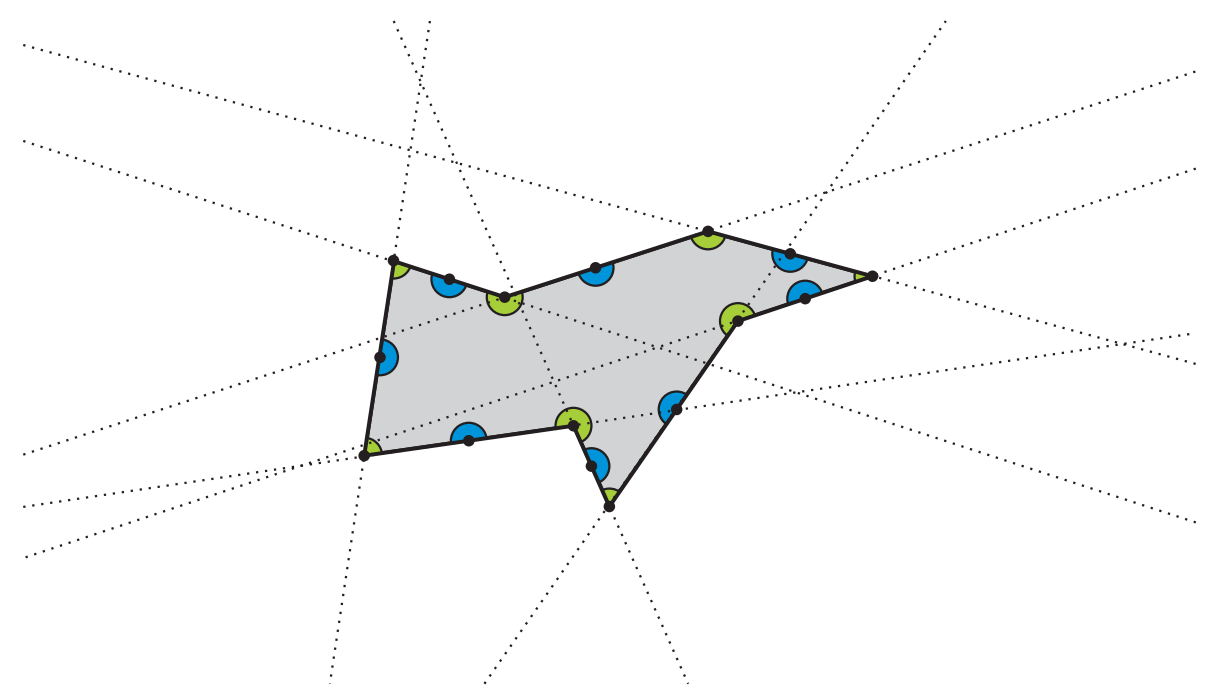

Figura 2. Parte da subdivisão planar induzida por antenas em posição natural.

as antenas em $G$, conforme a Figura 2. Uma discretização para o problema pode ser obtida a partir desta subdivisão planar. Considerando que a fórmula Booleana procurada deve ser satisfeita por todos os pontos internos ao polígono, mas não pelos externos, conforme demonstramos na dissertação, basta verificar esta propriedade em um único ponto de cada face da subdivisão planar.

Assim, a ideia inicial de nosso algoritmo foi criar um modelo PLI que busca minimizar a quantidade de antenas utilizadas, de tal forma que, com as antenas presentes na solução, seja possível encontrar uma fórmula Booleana válida para o problema. A partir desta formulação inicial, diversas melhorias foram sendo adicionadas ao método de solução durante o decorrer do trabalho. Tais melhorias ocorreram tanto no modelo PLI em si quanto na utilização de propriedades geométricas intrínsecas ao problema, como descreveremos brevemente na seção seguinte.

\section{Contribuições}

Nos capítulos 1 e 2 da dissertação, apresentamos uma discretização para o Problema do Posicionamento Natural de Antenas e, a partir dessa discretização, chegamos a um primeiro modelo PLI para resolvê-lo. Com este modelo inicial, mesmo realizando algumas otimizações na formulação matemática, só fomos capazes de encontrar soluções ótimas para instâncias com até 60 vértices, respeitado um tempo limite de 20 minutos de computação. A maior dificuldade surge por conta do grande número de faces na subdivisão planar resultante da discretização do problema, que tem forte impacto na quantidade de restrições do modelo.

Posteriormente, estudando as propriedades geométricas da discretização, definimos faces de sombra e luz: faces da subdivisão planar que representam as áreas de coberturas minimais e maximais em relação às antenas em posição natural. A obtenção destas faces foi realizada utilizando um algoritmo de varredura no plano, desenvolvido em conjunto com pesquisadores da Technische Universität Braunschweig (TUB), na Alemanha, durante o estágio de pesquisa no exterior realizado pelo mestrando. Através desta propriedade, pudemos reduzir a quantidade de regiões internas e externas que precisamos 
verificar ao resolver o problema em $98,9 \%$ em média.Tal redução traduz-se diretamente em um modelo matemático mais compacto e, portanto, mais fácil de ser calculado na prática. Com este decréscimo no tamanho da formulação, respeitando o mesmo limite de tempo de 20 minutos, conseguimos solucionar instâncias de até 100 vértices.

A partir dos resultados obtidos com as faces de sombra e luz, pareciam esgotadas as possibilidades de melhorar o desempenho computacional do algoritmo, a menos que houvesse uma quebra de paradigma. Como resultado desta observação, como pode ser visto no capítulo 4, propusemos um algoritmo iterativo em que vários modelos menores eram resolvidos a cada passo, até que, na última iteração, fosse possível concluir que a solução do modelo menor e a do original eram coincidentes. Com isso, foram resolvidas instâncias de 1.000 vértices em menos de 10 minutos, utilizando, em média, apenas 1,9\% total das restrições na última iteração, já considerando o modelo reduzido decorrente do uso das propriedades das faces de sombra e luz.

Finalmente, como pode ser visto no capítulo 5, com a inicialização do algoritmo iterativo com um subconjunto adequado das restrições, pudemos resolver todas as instâncias de 1.000 vértices em menos de seis minutos, inclusive casos envolvendo polígonos com buracos. Este ganho foi alcançado mediante um estudo cuidadoso em que procuramos identificar as relações de dominância entre as desigualdades lineares do modelo de programação inteira.

Os apêndices da dissertação mostram detalhes sobre o gerador de instâncias criado, bem como da ferramenta desenvolvida para editar, visualizar e resolver instâncias.

\section{Conclusão}

Nesta dissertação, descrevemos a pesquisa sobre o Problema do Posicionamento Natural de Antenas, onde o objetivo é minimizar o número de antenas em posição natural necessárias para descreverem o interior de um polígono. Graças a uma abordagem nãotrivial do problema em que utilizamos técnicas que possibilitaram ir além da resolução ingênua do modelo PLI, ao final deste trabalho, fomos capazes de resolver, na otimalidade, instâncias quase 20 vezes maiores do que aquelas tratadas no começo da pesquisa.

Para se atingir este resultado, foi feito um intenso trabalho experimental o qual foi composto de várias etapas. Ao término de cada uma destas etapas, seguiram-se cuidadosas análises das saídas das execuções, o que possibilitou uma melhoria contínua do algoritmo à medida que a pesquisa avançava. A exemplo do que já havia ocorrido com outras versões do problema de galeria de artes, novamente foi possível constatar a eficiência das técnicas de PLI na resolução exata de problemas de Geometria Computacional.

Evidentemente que o estudo do problema não se encerra com esta dissertação. Assim, visando contribuir com os pesquisadores interessados no tema, tornamos disponível publicamente o benchmark de instâncias que utilizamos em nossos experimentos (ver [Crepaldi et al. 2013b]). Como fornecemos não apenas os dados de entrada mas também as soluções ótimas de todas as instâncias, heurísticas, outras formulações PLI ou mesmo formas de soluções diferentes podem ser facilmente comparadas aos nossos resultados

Finalmente, destacamos os dois principais subprodutos da dissertação. O primeiro foi o trabalho "An Efficient Exact Algorithm for the Natural Wireless Localization Problem" que foi apresentado no CCCG 2013 - The 25th Canadian Conference on Com- 
putational Geometry (QUALIS B2), uma das mais importantes conferências na área de Geometria Computacional [Crepaldi et al. 2013a]. Após a participação no evento, fomos convidados a submeter uma versão completa do trabalho para uma edição de um volume especial do periódico CGTA - Computational Geometry: Theory and Applications (QUALIS A2), artigo este que já se encontra publicado [Crepaldi et al. 2015].

\section{Referências}

Borrmann, D., de Rezende, P. J., de Souza, C. C., Fekete, S. P., Friedrichs, S., Kröller, A., Nüchter, A., Schmidt, C., and Tozoni, D. C. (2013). Point guards and point clouds: Solving general art gallery problems. In Proceedings of the Twenty-ninth Annual Symposium on Computational Geometry, pages 347-348, New York. ACM.

Christ, T. and Hoffmann, M. (2009). Wireless localization with vertex guards is NP-hard. In 21st Canadian Conference on Computational Geometry, pages 149-152, Toronto.

Christ, T., Hoffmann, M., and Okamoto, Y. (2009). Natural wireless localization is NPhard. In Abstracts 25th European Workshop on Comp. Geometry, pages 175-178, Bruxelles.

Christ, T., Hoffmann, M., Okamoto, Y., and Uno, T. (2008). Improved bounds for wireless localization. In Algorithm Theory - SWAT 2008, volume 5124 of Lect. Notes in Comp. Science, pages 77-89, Goteborg. Springer Berlin Heidelberg.

Couto, M. C., de Souza, C. C., and de Rezende, P. J. (2008). Experimental evaluation of an exact algorithm for the orthogonal art gallery problem. In Experimental Algorithms, 7th International Workshop, WEA 2008, Provincetown, MA, USA, May 30-June 1, 2008, Proceedings, volume 5038 of Lect. Notes in Comp. Science, pages 101-113.

Couto, M. C., de Souza, C. C., and de Rezende, P. J. (2011). An exact algorithm for minimizing vertex guards on art galleries. Int. Trans. in Oper. Research, 18:425-448.

Crepaldi, B. E., de Rezende, P. J., and de Souza, C. C. (2013a). An efficient exact algorithm for the natural wireless localization problem. In 25th Canadian Conference on Computational Geometry, Waterloo, Ontario, August 8-10.

Crepaldi, B. E., de Rezende, P. J., and de Souza, C. C. (2013b). The Wireless Localization

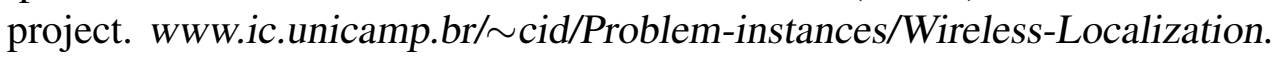

Crepaldi, B. E., de Rezende, P. J., and de Souza, C. C. (2015). Solving the natural wireless localization problem to optimality efficiently. Comp. Geom. and Appl., 48(5):370-379.

Dey, T. K. (1991). Triangulation and CSG representation of polyhedra with arbitrary genus. In Seventh Annual Symposium on Computational Geometry, pages 364-371.

Dobkin, D., Guibas, L., Hershberger, J., and Snoeyink, J. (1993). An efficient algorithm for finding the CSG representation of a simple polygon. Algorithmica, 10(1):1-23.

Eppstein, D., Goodrich, M. T., and Sitchinava, N. (2007). Guard placement for efficient point-in-polygon proofs. In 23rd Annual Symp. on Comp. Geom., pages 27-36.

O'Rourke, J. (1987). Art gallery theorems and algorithms. Oxford University Press, Inc., New York.

Urrutia, J. (2000). Art gallery and illumination problems. In Handbook of Computational Geometry, pages 973-1027. North-Holland. 